\title{
Everyday Discrimination in Adults with Knee Pain: The Role of Perceived Stress and Pain Catastrophizing
}

This article was published in the following Dove Press journal: Journal of Pain Research

\author{
Ellen L Terry' \\ $M$ Dottington Fullwood (D) ${ }^{2}$ \\ Staja Q Booker (D) \\ Josue $S$ Cardoso ${ }^{3}$ \\ Kimberly T Sibille ${ }^{2}$ \\ Toni L Glover (D) ${ }^{4}$ \\ Kathryn A Thompson ${ }^{5}$ \\ Adriana S Addison ${ }^{6}$ \\ Burel R Goodin (iD) ${ }^{5}$ \\ Roland Staud (iD ${ }^{7}$ \\ Laura B Hughes ${ }^{6}$ \\ Laurence A Bradley ${ }^{6}$ \\ David T Redden ${ }^{8}$ \\ Emily J Bartley ${ }^{9}$ \\ Roger B Fillingim ${ }^{3,9}$
}

'Department of Biobehavioral Nursing Science, University of Florida, Gainesville, Florida, United States; ${ }^{2}$ Department of Aging and Geriatric Research, University of Florida, Gainesville, Florida, United States; ${ }^{3}$ Pain Research and Intervention Center of Excellence (PRICE), Gainesville, Florida, United States; ${ }^{4}$ Oakland University, School of Nursing, Rochester, MI, United States; ${ }^{5}$ Department of Psychology, University of Alabama at Birmingham, Birmingham, Alabama, United States; ${ }^{6}$ University of Alabama at Birmingham, Division of Clinical Immunology \& Rheumatology, Birmingham, Alabama, United States; ${ }^{7}$ Department of Medicine, University of Florida, Gainesville, Florida, United States; ${ }^{8}$ Department of Biostatistics, University of Alabama at Birmingham, Birmingham, Alabama, United States; ' University of Florida, Community Dentistry and Behavioral Science, Gainesville, Florida, United States

Correspondence: Ellen L Terry Department of Biobehavioral Nursing Science, University of Florida, PO Box I00197, Gainesville FL 32610-0197 Tel (+352) 273-644I

Email elterry@ufl.edu
Purpose: Research indicates pain-related disparities in the impact of knee osteoarthritis (OA) across both sex and ethnicity/race. While several factors likely contribute to these disparities, experiences of discrimination are associated with poor OA-related pain, disability, and functional performance. However, the mechanisms that mediate experiences of discrimination and OA-related outcomes are unclear. The current cross-sectional study examined the associations between everyday experiences of discrimination and clinical pain, disability and functional performance among non-Hispanic Black (NHB) and non-Hispanic White (NHW) persons with or at risk of knee OA and assessed the serial mediated model of perceived stress and pain catastrophizing on these relationships in women only.

Patients and Methods: Participants were 188 community-dwelling adults who presented with unilateral or bilateral knee pain and screened positive for clinical knee pain. Participants completed several measures including experiences of discrimination, Perceived Stress Scale, Coping Strategies Questionnaire-Revised (CSQ-R): Pain Catastrophizing subscale, Western Ontario and McMaster Universities Osteoarthritis Index (WOMAC), Graded Chronic Pain Scale (GCPS), and Short Physical Performance Battery (SPPB).

Results: As compared to NHW participants, NHB individuals reported experiencing significantly higher levels of discrimination $(F(1,175)=26.660, p<0.001)$, greater levels of pain catastrophizing $(F(1,180)=12.919, p<0.001)$, higher levels of clinical pain and disability, and lower levels of physical function $(p \mathbf{s}<0.05)$. However, perceived stress was positively correlated with discrimination in the NHW group only (NHW females: $r=0.40, p<0.01$; NHW males: $r=0.37, p<0.05)$. Further, perceived stress and pain catastrophizing mediated the relationship between discrimination and outcome variables (WOMAC pain, GCPS interference [pain disability], and SPPB function) in female participants after controlling for relevant sociodemographic variables (study site, age, race, income, and body mass index).

Conclusion: These results may have implications for the treatment of perceived stress and catastrophizing as a means to reduce the negative impact of experiences of discrimination on the experience of chronic pain, particularly for women.

Keywords: experiences of discrimination, Perceived Stress Scale, pain catastrophizing, knee osteoarthritis, pain, disability, physical function

\section{Introduction}

Osteoarthritis (OA) is a leading cause of musculoskeletal pain and disability, affecting over $13 \%$ of the United States (US) adult population. ${ }^{1,2}$ OA prevalence in the US has doubled between 1999 and 2014 and is more common than other forms of arthritis, including rheumatoid arthritis, which has seen a decline over the 
same period. ${ }^{3}$ While OA is a disease that can affect any synovial joint, the knee is among the most frequently affected joint. ${ }^{4}$ Knee OA produces substantial physical (ie, knee pain, reduced function), psychological (ie, increased stress, poorer quality of life) and socioeconomic (ie, increased healthcare utilization, surgical intervention) burden..$^{5-7}$

Research evidence indicates disparities in the impact of knee OA across both sex and ethnicity/race. Specifically, the lifetime risk of symptomatic OA is higher in women (16.3\%) compared to men $(11.4 \%),{ }^{8}$ thus, the prevalence of radiographic and symptomatic knee OA is greater in women compared to men. ${ }^{9,10}$ Furthermore, women report greater pain severity $^{9,11}$ and women with radiographic symptomatic knee OA have a higher incidence of total knee replacement, although this trend in total knee replacement is predominately driven by non-Hispanic White (NHW) women compared to women of other ethnic/racial groups. ${ }^{12}$ Therefore, it is important to understand disparities-related factors that contribute to knee OA pain in women. Regarding ethnicity/race, a recent meta-analysis provides compelling evidence that ethnic/racial minorities, specifically non-Hispanic Black (NHB) individuals, experience greater pain and disability compared to their NHW counterparts. ${ }^{13}$ Specifically, NHBs report greater pain severity, ${ }^{14,15}$ higher levels of pain-related physical and psychosocial disability, ${ }^{16}$ and more severe functional limitations. ${ }^{17,18}$

The greater burden of knee OA among women and NHB individuals raises the question of whether common factors may contribute to OA-related pain and disability in these high-risk groups. While a multitude of factors likely contribute to the growing disparities of pain in women with knee OA, Luong et al ${ }^{19}$ identified several psychosocial mediators (eg, perceived discrimination, catastrophizing) that may influence OA-related outcomes. Indeed, experiences of discrimination could be an important contributor to health disparities in women and individuals from racial/ethnic minority groups. ${ }^{20,21}$ Discrimination involves the behavioral demonstration of bias against a specific group. ${ }^{22}$ Exposure to discrimination may be a chronic stressor that results in dysregulation of biopsychosocial processes ${ }^{23}$ increasing the susceptibility to the development of chronic pain. Experiences of discrimination are associated with chronic life stress, ${ }^{24}$ greater experimental pain, ${ }^{25}$ chronic pain, ${ }^{26-28}$ and disability. ${ }^{17}$ Also, pain catastrophizing is associated with greater stress and poorer OA-related pain outcomes in women. ${ }^{29}$ Waugh and colleagues ${ }^{30}$ provide evidence that internalized stigma in persons with chronic pain was associated with higher levels of pain catastrophizing, which negatively impacted individuals' sense of control over pain. Therefore, experiences of discrimination, which can result in increased internalized stigma, could lead to increased pain catastrophizing, which can facilitate pain. However, no study has investigated the extent to which perceived stress and pain catastrophizing may mediate the association of discrimination with pain-related outcomes.

This gap in knowledge represents an opportunity to identify important psychosocial determinants of OA pain that can potentially be mitigated and/or reduced to improve OA outcomes. The current study aims to examine whether perceived stress and pain catastrophizing serially mediate the relationship between experiences of discrimination and OA-related pain, disability, and functional impairment in a racially diverse sample of adults with knee pain. We tested the following hypotheses: Hypothesis 1: Higher rates of perceived discrimination in NHB participants would be associated with higher rates of perceived stress, pain catastrophizing, clinical pain and disability, and lower levels of physical function compared to NHW participants. Hypothesis 2: Perceived stress and pain catastrophizing would serially mediate the association of discrimination with clinical pain, disability, and physical function in women.

\section{Patients and Methods Study Overview}

The current study was a cross-sectional study of a larger ongoing longitudinal observational cohort study titled Understanding Pain and Limitations in Osteoarthritic Disease - Second Cycle (UPLOAD-2) that aims to elucidate the mechanisms underlying ethnic/racial group differences in knee pain among adults with or at risk for knee OA. The UPLOAD-2 study was a multi-site investigation being conducted at the University of Florida and the University of Alabama at Birmingham. The participants described in the current analysis were recruited at both sites between August 2015 and May 2017. All participants provided written informed consent, and this study was conducted in accordance with the Declaration of Helsinki.

\section{Participants}

Participants were 188 (117 women, 71 men) communitydwelling adults between 45 and 85 years of age who self-identified as NHB or NHW, presented with unilateral or bilateral knee pain and screened positive for clinical knee OA. ${ }^{31}$ 


\section{Procedures}

All procedures were reviewed and approved by the Institutional Review Boards at the University of Florida and the University of Alabama at Birmingham. Participants completed a standardized telephone screening to confirm initial eligibility. The following sociodemographic and physical health data were acquired as part of the screening to determine initial eligibility: self-reported sex, age, ethnic/ racial identity, and a brief health history including symptoms of knee OA. This screening questionnaire used to determine symptoms of knee OA showed $87 \%$ sensitivity and $92 \%$ specificity for radiographically confirmed symptomatic knee OA. ${ }^{32}$ All participants were negative for other rheumatologic conditions including rheumatoid arthritis, fibromyalgia, which could explain knee pain. Given the broad definition of $\mathrm{OA}^{33}$ we adopted this approach to be as inclusive as possible in recruitment, as our primary focus is on understanding factors associated with knee pain rather than OA pathophysiology itself. Moreover, because this prospective observational study is designed to evaluate the progression of OA-related symptoms, we sought to enroll a cohort with a broad range of OA characteristics, from very early signs to advanced disease.

Participants who met the inclusion criteria were brought into the laboratory and were consented prior to data collection. Participants then completed health and pain history questionnaires, which included sociodemographic questions (eg, race/ethnicity, income), and body mass index (BMI) measurements were obtained. Prior to the end of the first visit, participants completed the Short Physical Performance Battery (SPPB). Questionnaires assessing experiences of discrimination, psychosocial variables, clinical pain, and disability were administered electronically via email prior to the subsequent visits of this multisession protocol (ie, three laboratory visits) to reduce participant burden. However, if the participant did not have access to a computer to complete the questionnaires electronically, the questionnaires were completed at the beginning of the next laboratory visit. Participants completed several questionnaires (ie, CSQ-R pain catastrophizing subscale, WOMAC pain, GCPS interference, and Perceived Stress Scale) prior to or at the beginning of visit two, and the Experiences of Discrimination questionnaire was completed prior to or at the beginning of visit three.

Participants were recruited through the community via multiple advertisement methods (eg, posted fliers) and via clinic-based methods. Participants were excluded for the following self-reported conditions: 1) prosthetic knee replacement or other clinically significant surgery to the arthritic knee; 2) uncontrolled hypertension; 3) heart disease; 4) peripheral neuropathy; 5) systemic rheumatic disorders 6) neurological diseases 7) significantly greater pain in body sites other than in the knee; 8) daily opioid use; 9) hospitalization within the preceding year for psychiatric illness; or 10) pregnant or nursing.

\section{Measures \\ Outcome Variables}

Western Ontario and McMaster Universities Osteoarthritis Index (WOMAC)

The WOMAC is a reliable (Cronbach's alpha $\geq 0.80$ ) and well-validated measure of lower extremity pain in persons with OA. ${ }^{34,35}$ WOMAC assesses symptoms of knee OA in the past 48 hours. The WOMAC pain (range 0-20) subscale was included in data analysis. Higher scores on this subscale indicate greater pain.

\section{Graded Chronic Pain Scale (GCPS)}

The GCPS is a 7-item scale that evaluates pain-related interference over the past 6 months. The GCPS yields an overall "Disability" score. ${ }^{36}$ With a $0-10$ NRS, participants rated the degree to which their knee pain interfered with daily activities including recreation and work during the past 6 months. These 3 items were averaged and multiplied by 10 to generate a disability score, with higher scores indicating greater symptomatology.

\section{Short Physical Performance Battery (SPPB)}

The SPPB is a standardized measure of lower-extremity function that includes three performance tests: standing balance, 4-meter gait speed, and chair-rising tasks. ${ }^{37}$ Each measure is scored from 0 (worst performance) to 4 (best performance), and a total score ranging from 0 to 12 is calculated by summing the scores from all three subscales. Lower scores indicating poorer function. The total score was used in the analyses.

\section{Independent Variable}

\section{Experiences of Discrimination (EOD)}

The EOD assessed experiences of discrimination across 9 different situations. ${ }^{20}$ Participants indicated the frequency to which they experienced various forms of day-to-day mistreatment (never, once, two or three times, four or more times), and for endorsed experiences participants also indicated the reason for the mistreatment (eg, race, 
gender, weight, ... ). Examples include, "You have been treated with less respect than other people," "You have been called names or insulted" and "You have been threatened or harassed." These items are framed without reference to race, ethnicity, or gender.

\section{Mediator Variables}

\section{Perceived Stress Scale}

Perceived stress was assessed through the 10-item Perceived Stress Scale (PSS), a reliable (Cronbach's alpha $\geq 0.84$ ) and valid scale designed to measure the role of nonspecific appraised stress. ${ }^{38,39}$ Participants are asked to rate ( $0=$ "never" to $4=$ "very often") statements asking about thoughts and feelings over the past month. A total perceived stress score is computed.

\section{Coping Strategies Questionnaire-Revised (CSQ-R)}

The CSQ-R Pain Catastrophizing subscale assessed the helplessness dimension of catastrophizing. The reliability and validity of the CSQ-R subscales have previously been shown to be acceptable. ${ }^{40,41}$

\section{Data Analysis}

All data were analyzed using SPSS 25.0 (IBM, Chicago, IL), and data were checked for normality, outliers, and missing values. Sex differences in sociodemographic, clinical characteristics and experiences of discrimination were assessed using chi-square for dichotomous variables and independent samples t-tests for continuous variables. Pearson correlations were conducted to examine associations between measures of experiences of discrimination and psychosocial, clinical pain, disability, and functional performance variables. A two-way between-groups analysis of covariance (ANCOVA) was conducted to assess differences across race and sex in experiences of discrimination and outcome variables (psychosocial, clinical pain, disability, and functional performance). Covariates included study site, age, and BMI. Hayes' PROCESS macro $^{42}$ was used to examine the serial multiple mediator model. Serial mediation assumes one mediator affects another mediator within a specified direction such that experiences of discrimination could increase perceived stress which could increase pain catastrophizing resulting in poor clinical and functional outcomes (ie, experiences of discrimination perceived stress pain catastrophizing clinical and functional outcomes). The total effect denoted as $c$ in the model predicts the outcome $(\mathrm{Y})$ from experiences of discrimination (X), adjusting for covariates, but excluding the mediating variables (perceived stress $\left[M_{1}\right]$ and pain catastrophizing $\left[M_{2}\right]$ ). The total effect consists of three indirect effects $\left(a_{1} b_{1}, a_{1} d_{21} b_{2}, a_{1} b_{2}\right)$ and one direct effect $\left(c^{\prime}\right) \cdot{ }^{43}$ Further, a bootstrapping procedure was used to conduct inference tests for indirect effects. ${ }^{43}$ The $95 \%$ bias-corrected bootstrap confidence interval is based on 10,000 bootstrap samples to generate the path estimates and the indirect effects. Results were statistically significant when zero was not contained in the $95 \%$ confidence interval. Serial mediation analyses were conducted in female participants only because experiences of discrimination were not associated with the outcome variables among NHB males. As a sensitivity analysis, serial mediation analyses stratified by race were conducted in female participants, which showed similar results compared to the combined analysis. Therefore, the analyses presented below include the combined sample to increase statistical power. Study site, age, ethnic/race group, income, and BMI were included as covariates in all mediation analyses. Significance was set at $p<0.05$ (two-tailed).

\section{Results}

\section{Participant Characteristics}

The sociodemographic, clinical, and anthropometric data of the participants overall and by race and sex are shown in Table 1. Importantly, there were no differences in knee pain duration between the groups. Females outnumbered male participants. NHB female participants were younger, had lower income and higher BMI than NHW female participants. Additionally, more NHW female participants were married and retired compared to NHB female participants.

As shown in Table 2, there were no statistical race group differences in frequency of experiences of discrimination among female participants. NHB males reported higher experiences of discrimination due to race and there was a statistically significant difference in the number of experiences of discrimination between NHB male and NHW male participants.

\section{Group Differences in Outcome and Mediator Variables}

NHB participants reported more experiences of discrimination compared to NHWs, $F(1,175)=26.660, p<0.001$ and this race group difference was larger among males than females (raceXsex interaction), $F(1,175)=6.214$, $p=0.014$ (see Table 3). No race or sex differences 
Table I Sociodemographic and Clinical Characteristics of Participants Across Sex and Race

\begin{tabular}{|c|c|c|c|c|c|c|}
\hline & $\begin{array}{l}\text { NHB Females } \\
(N=59)\end{array}$ & $\begin{array}{l}\text { NHW Females } \\
(\mathrm{N}=\mathbf{5 8})\end{array}$ & $p$ & $\begin{array}{l}\text { NHB Males } \\
(\mathrm{N}=39)\end{array}$ & $\begin{array}{l}\text { NHW Males } \\
(\mathrm{N}=32)\end{array}$ & $p$ \\
\hline & M or $\mathbf{N}($ SD or \%) & $M$ or $N(S D$ or $\%)$ & & M or N (SD or \%) & $M$ or $\mathbf{N}(\mathrm{SD}$ or $\%)$ & \\
\hline Age (years)* & $55.4(6.9)$ & $59.2(8.6)$ & 0.01 & $58.0(5.6)$ & $60.4(9.1)$ & 0.20 \\
\hline $\begin{array}{l}\text { Sex } \\
\quad \text { Female } \\
\text { Male }\end{array}$ & $\begin{array}{l}59(50.4) \\
-\end{array}$ & $\begin{array}{l}58(49.6) \\
-\end{array}$ & & $\begin{array}{l}- \\
39(54.9)\end{array}$ & $\begin{array}{l}- \\
32(45.1)\end{array}$ & \\
\hline $\begin{array}{l}\text { Race } \\
\text { Non-Hispanic black } \\
\text { Non-Hispanic white }\end{array}$ & $\begin{array}{l}59(50.4) \\
-\end{array}$ & $\begin{array}{l}- \\
58(49.6)\end{array}$ & & $\begin{array}{l}39(54.9) \\
-\end{array}$ & $\begin{array}{l}- \\
32(45.1)\end{array}$ & \\
\hline 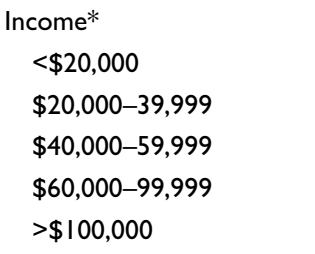 & $\begin{array}{l}27(48.2) \\
15(26.8) \\
4(7.1) \\
8(14.3) \\
2(3.6)\end{array}$ & $\begin{array}{l}14(24.6) \\
7(12.3) \\
15(26.3) \\
10(17.5) \\
11(19.3)\end{array}$ & $<0.01$ & $\begin{array}{l}25(64.1) \\
7(17.9) \\
4(10.3) \\
2(5.1) \\
1(2.6)\end{array}$ & $\begin{array}{l}13(40.6) \\
5(15.6) \\
6(18.8) \\
5(15.6) \\
3(9.4)\end{array}$ & 0.19 \\
\hline $\begin{array}{l}\text { Education } \\
\text { Some high school } \\
\text { High school degree } \\
\text { Associates or bachelors } \\
\text { Graduate/Professional }\end{array}$ & $\begin{array}{l}5(8.5) \\
28(47.5) \\
19(32.2) \\
7(11.9)\end{array}$ & $\begin{array}{l}1(1.7) \\
19(32.8) \\
25(43.1) \\
13(22.4)\end{array}$ & 0.07 & $\begin{array}{l}5(12.8) \\
19(48.7) \\
13(33.3) \\
2(5.1)\end{array}$ & $\begin{array}{l}2(6.3) \\
12(37.5) \\
13(40.6) \\
5(15.6)\end{array}$ & 0.32 \\
\hline $\begin{array}{l}\text { Marital Status* } \\
\text { Married } \\
\text { Not married }\end{array}$ & $\begin{array}{l}13(22.4) \\
45(77.6)\end{array}$ & $\begin{array}{l}30(51.7) \\
28(48.3)\end{array}$ & $<0.01$ & $\begin{array}{l}\mathrm{II}(28.9) \\
27(7 \mathrm{I} . \mathrm{I})\end{array}$ & $\begin{array}{l}13(40.6) \\
19(59.4)\end{array}$ & 0.44 \\
\hline $\begin{array}{l}\text { Employment* } \\
\text { Employed } \\
\text { Not employed } \\
\text { Retired } \\
\text { BMl }\left(\mathrm{kg} / \mathrm{m}^{2}\right)^{*}\end{array}$ & $\begin{array}{l}27(65.9) \\
8(19.5) \\
6(14.6) \\
35.6(8.1)\end{array}$ & $\begin{array}{l}25(49.0) \\
6(11.8) \\
20(39.2) \\
31.4(8.0)\end{array}$ & 0.01 & $\begin{array}{l}13(48.1) \\
6(22.2) \\
8(29.6) \\
29.04(5.7)\end{array}$ & $\begin{array}{l}8(32.0) \\
6(24.0) \\
11(44.0) \\
30.3(5.9)\end{array}$ & $\begin{array}{l}0.45 \\
0.37\end{array}$ \\
\hline $\begin{array}{l}\text { Knee Pain Duration } \\
\quad<1 \text { year } \\
1-5 \text { years } \\
>5 \text { years }\end{array}$ & $\begin{array}{l}10(16.9) \\
29(49.2) \\
20(33.9)\end{array}$ & $\begin{array}{l}8(14.0) \\
21(36.8) \\
28(49.1)\end{array}$ & 0.25 & $\begin{array}{l}4(10.3) \\
15(38.5) \\
20(51.3)\end{array}$ & $\begin{array}{l}3(9.4) \\
9(28.1) \\
20(62.5)\end{array}$ & 0.62 \\
\hline
\end{tabular}

Note: $*_{p}<0.05$.

Abbreviation: BMI, body mass index.

emerged for perceived stress $(p s>0.50)$. NHBs reported higher levels of pain catastrophizing compared to NHWs, $F(1,180)=12.919, p<0.001$.

NHB participants reported higher rates of WOMAC pain compared to NHW participants, $F(1,180)=10.059$, $p=0.002$. NHB individuals reported higher rates of GCPS interference (pain disability) than NHW participants, $F(1,181)=13.953, p<0.001$. In addition, NHB participants showed poorer functional performance on the SPPB than NHW participants, $F(1,181)=9.005, p=0.003$.

\section{Pearson Correlations}

Table 4 presents zero-order correlations of experiences of discrimination with psychosocial variables, clinical pain, disability, and functional outcomes by race and sex. For NHB females, pain catastrophizing and SPPB functional performance were correlated with experiences of discrimination. Perceived stress, pain catastrophizing, and WOMAC pain were associated with experiences of discrimination in NHW females. Interestingly, experiences of discrimination were not 
Table 2 Experiences of Discrimination by Race and Sex

\begin{tabular}{|c|c|c|c|c|c|c|}
\hline & $\begin{array}{l}\text { NHB Females } \\
(N=59) N(\%)\end{array}$ & $\begin{array}{l}\text { NHW Females } \\
(N=58) \mathbf{N}(\%)\end{array}$ & $\mathbf{p}$ & $\begin{array}{l}\text { NHB Males } \\
(\mathbf{N}=39) \\
\text { N (\%) }\end{array}$ & $\begin{array}{l}\text { NHW Males } \\
(\mathbf{N}=32) \mathbf{N}(\%)\end{array}$ & $\mathbf{p}$ \\
\hline Experiences of discrimination & & & 0.32 & & & $0.03 *$ \\
\hline Ancestry or national origins & $2(3.7)$ & - & & $0(0.0)$ & - & \\
\hline Gender & I (I.9) & $14(29.2)$ & & $0(0.0)$ & $\mathrm{I}(4.5)$ & \\
\hline Race & $30(55.6)$ & $\mathrm{I}(2.1)$ & & $29(80.6)$ & $3(13.6)$ & \\
\hline Religion & $0(0.0)$ & $\mathrm{I}(2.1)$ & & I (2.8) & $3(13.6)$ & \\
\hline Height or weight & I (I.9) & $10(20.8)$ & & $0(0.0)$ & I (4.5) & \\
\hline Shade of skin color & $7(13.0)$ & $I(2.1)$ & & $2(5.6)$ & $0(0.0)$ & \\
\hline Sexual orientation & $2(3.7)$ & I (2.I) & & I (2.8) & $0(0.0)$ & \\
\hline Education or income level & $4(7.4)$ & $2(4.2)$ & & I (2.8) & $2(9.1)$ & \\
\hline Physical disability & $3(5.6)$ & I (2.I) & & $0(0.0)$ & $2(9.1)$ & \\
\hline Other unspecified reason & $4(7.4)$ & $17(35.4)$ & & $2(5.6)$ & $10(45.5)$ & \\
\hline
\end{tabular}

Note: ${ }^{*} p<0.05$.

Abbreviations: NHB, non-Hispanic black; NHW, non-Hispanic white.

Table 3 Inferential Statistics for Experiences of Discrimination, Psychosocial Variables, Clinical Pain, Disability, and Functional Outcomes by Race and Sex

\begin{tabular}{|l|l|l|l|l|}
\hline & NHB Females (N = 59) & NHW Females (N = 58) & NHB Males (N = 39) & NHW Males (N = 32) \\
\cline { 2 - 5 } & M(SD) & M(SD) & M(SD) & M(SD) \\
\hline Experiences of discrimination*** & $19.07(12.54)$ & $13.94(12.50)$ & $24.58(12.73)$ & $10.04(10.52)$ \\
Perceived stress & $14.32(7.57)$ & $13.87(6.76)$ & $14.08(5.47)$ & $13.98(6.70)$ \\
Pain catastrophizin** & $1.61(1.36)$ & $0.97(1.13)$ & $1.71(1.33)$ & $1.07(1.21)$ \\
WOMAC pain* & $8.39(3.94)$ & $6.59(4.47)$ & $9.32(4.12)$ & $7.25(3.81)$ \\
Pain disability* & $53.38(30.02)$ & $37.97(31.32)$ & $57.63(24.39)$ & $40.87(27.14)$ \\
SPPB function* & $9.15(1.81)$ & $9.59(1.73)$ & $8.90(1.77)$ & $9.93(1.16)$ \\
\hline
\end{tabular}

Notes: ${ }^{*} p<0.05$ main effect of race, ${ }^{* *} p<0.05$ interaction of race and sex. Analyses were adjusted for the following covariates: study site, age, and body mass index. Abbreviations: WOMAC, Western Ontario and McMaster Universities Osteoarthritis Index; SPPB, Short Physical Performance Battery.

associated with any outcome variables in NHB males. Lastly, only perceived stress was positively associated with experiences of discrimination in NHW males.

Table 4 Zero-Order Correlations Between Psychosocial Variables, Clinical Pain, Disability, and Functional Outcomes and Experiences of Discrimination by Race and Sex

\begin{tabular}{|l|l|l|l|l|}
\hline \multirow{2}{*}{} & \multicolumn{4}{|l|}{ Experiences of Discrimination } \\
\cline { 2 - 5 } & NHB & NHW & NHB & NHW \\
& Females & Females & Males & Males \\
& $\mathbf{( N = 5 9 )}$ & $\mathbf{( N = 5 8 )}$ & $\mathbf{( N = 3 9 )}$ & $\mathbf{( N = 3 2 )}$ \\
\hline Perceived stress & 0.23 & $0.40^{* *}$ & -0.08 & $0.37^{*}$ \\
Pain catastrophizing & $0.30^{*}$ & $0.32^{*}$ & -0.04 & 0.04 \\
WOMAC pain & 0.12 & $0.42^{* *}$ & -0.01 & 0.19 \\
Pain disability & 0.26 & 0.23 & -0.07 & 0.11 \\
SPPB function & $-0.27^{*}$ & -0.20 & -0.03 & -0.16 \\
\hline
\end{tabular}

Notes: $* p<0.05 ; * * p<0.01$.

Abbreviations: NHB, non-Hispanic black; NHW, non-Hispanic white; WOMAC, Western Ontario and McMaster Universities Osteoarthritis Index; SPPB, Short Physical Performance Battery.

\section{Mediation Analysis}

Due to the lack of correlation between experiences of discrimination and outcome variables in the male participants, we conducted mediation analyses in the female participants only. Data from the serial mediation analyses stratified by race in female participants were not included in the manuscript as the pattern of results was similar to that from the overall sample. Therefore, females were combined in the serial mediation analyses to increase statistical power.

\section{WOMAC Pain}

The total effect, $c$, of experiences of discrimination $(\mathrm{X})$ on WOMAC pain (Y) after controlling for covariates was 0.0660 , indicating higher levels of discrimination predict higher WOMAC pain (Figure 1A and Table 5). The direct effect, $c^{\prime}$, was not statistically significant, suggesting that experiences of discrimination were unrelated to WOMAC pain, independent of the effect of stress and trait 


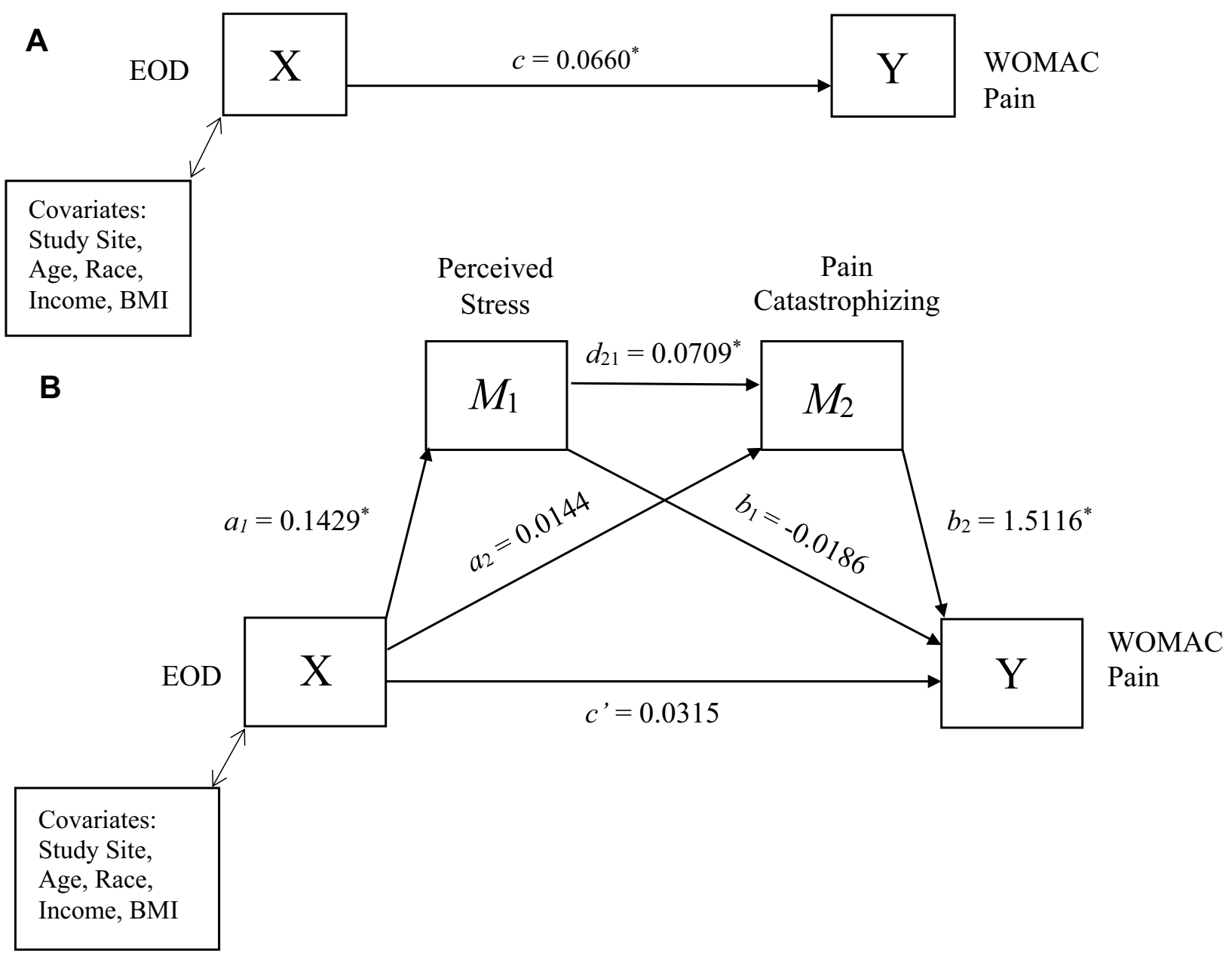

Figure I Path diagram showing (A) the total effect of EOD on WOMAC Pain and (B) the direct effect and causal paths linking EOD to WOMAC Pain. *Statistical significance (the bootstrap confidence interval is above or below zero).

Abbreviations: EOD, Everyday discrimination; WOMAC Pain, The Western Ontario and McMaster Universities Osteoarthritis Index; BMI, body mass index.

catastrophizing (Figure 1B and Table 5). Furthermore, the indirect effect $\left(a_{1} b_{1}\right)$ of discrimination on WOMAC pain through perceived stress was not significant. However, the indirect effect of discrimination through perceived stress and pain catastrophizing in serial, with perceived stress modeled as affecting pain catastrophizing, which in turn influences WOMAC pain $\left(a_{1} d_{21} b_{2}\right)$ was significant ( 0.0043 to 0.0386 ). Those who experienced high discrimination experienced greater perceived stress $\left(a_{1}=0.1429\right)$, which was associated with greater levels of pain catastrophizing $\left(d_{21}=0.0709\right)$, and predicted greater WOMAC pain $\left(b_{2}=1.5116\right)$. The indirect effect $\left(a_{2} b_{2}\right)$ of discrimination on WOMAC pain through trait pain catastrophizing was significant (0.0008 to 0.0509). Experiences of discrimination were related to greater levels of pain catastro- phizing $\left(a_{2}=0.0144\right)$, which in turn was associated with greater WOMAC pain $\left(b_{2}=1.5116\right)$.

\section{GCPS Interference}

The total effect, $c$, of experiences of discrimination (X) on interference $(\mathrm{Y})$ after controlling for covariates was not statistically significant, suggesting higher levels of discrimination were unrelated to interference (Figure $2 \mathrm{~A}$ and Table 6 ). The direct effect, $c^{\prime}$, was not statistically significant, suggesting that experiences of discrimination were unrelated to interference, independent of the effect of stress and trait catastrophizing (Figure 2B and Table 6). However, the indirect effect $\left(a_{1} b_{1}\right)$ of discrimination on interference through perceived stress was significant, suggesting that those who experience higher levels of discri- 
Table 5 Path Coefficients, Indirect Effects and 95\% BiasCorrected. Bootstrap Confidence Intervals from OLS Regression Predicting Score on WOMAC Pain $(\mathrm{N}=107)$

\begin{tabular}{|l|l|l|l|}
\hline \multirow{2}{*}{ Path } & \multirow{2}{*}{ Estimate } & \multicolumn{2}{|l|}{$\mathbf{9 5 \%}$ Cl } \\
\cline { 3 - 4 } & & Lower & Upper \\
\hline Total effect $(c)$ & $0.0660^{*}$ & 0.0026 & 0.1294 \\
Direct effect $\left(c^{\prime}\right)$ & 0.0315 & -0.0288 & 0.0918 \\
$a_{1}$ (EOD $\rightarrow$ Perceived Stress) & $0.1429^{*}$ & 0.0372 & 0.2486 \\
$a_{2}$ (EOD $\rightarrow$ Pain Catastrophizing) & 0.0144 & -0.0037 & 0.0325 \\
$d_{21 \text { (Perceived Stress } \rightarrow \text { Pain Catastrophizing) }}$ & $0.0709^{*}$ & 0.0381 & 0.1037 \\
$b_{1}$ (Perceived Stress $\rightarrow$ WOMAC pain) & -0.0186 & -0.1361 & 0.0989 \\
$b_{2}$ (Pain Catastrophizing $\rightarrow$ WOMAC pain) & $1.5116^{*}$ & 0.8556 & 2.1676 \\
\hline Indirect Effects & & & \\
$a_{1} b_{1}$ & -0.0027 & -0.0263 & 0.0112 \\
$a_{1} d_{21} b_{2}$ & $0.0153^{*}$ & 0.0043 & 0.0386 \\
$a_{2} b_{2}$ & $0.0218^{*}$ & 0.0008 & 0.0509 \\
Total indirect effect & $0.0345^{*}$ & 0.0080 & 0.0734 \\
\hline
\end{tabular}

Notes: *Statistical significance (the bootstrap confidence interval is above or below zero). Models include controls for site, age, race, income, and body mass index.

mination reported greater perceived stress $\left(a_{1}=0.1431\right)$, which predicted greater interference $\left(b_{1}=0.8620\right)$. Furthermore, the indirect effect of discrimination through perceived stress and pain catastrophizing in serial, with perceived stress modeled as affecting pain catastrophizing, which in turn influences interference $\left(a_{1} d_{21} b_{2}\right)$ was significant $(0.0226$ to 0.2312$)$. Those who experience high discrimination experienced greater perceived stress $\left(a_{1}=\right.$ $0.1431)$, which was associated with greater levels of pain catastrophizing $\left(d_{21}=0.0713\right)$, and which predicted greater interference $\left(b_{2}=8.5110\right)$. The indirect effect $\left(a_{2}\right.$ $b_{2}$ ) of discrimination on interference through trait pain catastrophizing was significant (0.0007 to 0.3096). Experiences of discrimination lead to greater levels of pain catastrophizing $\left(a_{2}=0.0144\right)$, which in turn was associated with greater levels of interference $\left(b_{2}=8.5110\right)$.

\section{SPPB Total Function}

The total effect, $c$, of experiences of discrimination $(\mathrm{X})$ on functional performance $(\mathrm{Y})$ after controlling for covariates was -0.0149 , indicating higher levels of discrimination predict poorer functional performance (Figure $3 \mathrm{~A}$ and Table 7). The direct effect, $c^{\prime}$, was not statistically significant, suggesting that experiences of discrimination were unrelated to functional performance, independent of the effect of stress and trait catastrophizing (Figure 3B and Table 7). However, the indirect effect $\left(a_{1} b_{1}=0.1431\right.$ $(-0.0473)=-0.0068)$ of discrimination on functional performance through perceived stress was significant, suggesting those who experienced high levels of discrimination reported higher levels of perceived stress, which predicted poorer functional performance. Further, the indirect effect of discrimination through perceived stress and pain catastrophizing in serial, with perceived stress modeled as affecting pain catastrophizing, which in turn influences functional performance $\left(a_{1} d_{21} b_{2}\right.$ $=0.1431(0.0713)-0.2693=-0.0027))$ was significant $(-0.0093$ to -0.0002$)$. Those who experienced high discrimination experienced greater perceived stress, which was associated with higher levels of pain catastrophizing, and predicted poorer functional performance. The indirect effect $\left.\left(a_{2} b_{2}=0.0144(-0.2693)=-0.0039\right)\right)$ of discrimination on functional performance through trait pain catastrophizing was not significant ( -0.0126 to 0.0002$)$.

\section{Discussion}

This is the first study to examine this serial mediation model and to assess discrimination, perceived stress and catastrophizing as a potential common pathway contributing to greater pain burden among NHW and NHB women with or at risk of knee OA. Accumulating evidence has documented that experiences of discrimination are associated with poorer pain-related outcomes, ${ }^{17,25,27,28,44}$ as well as adverse psychosocial outcomes such as psychological distress, ${ }^{26}$ perceived stress, ${ }^{24}$ and pain catastrophizing. ${ }^{30}$ Much of the evidence on the deleterious effects of perceived discrimination in pain outcomes have focused on racial discrimination. ${ }^{27,28,45}$ The current study adds to the literature by examining whether psychosocial mediators (ie, perceived stress, pain catastrophizing) are mechanisms by which everyday experiences of discrimination, beyond racial discrimination, influence pain-related and functional outcomes.

Overall, Hypothesis 1 was not supported by the findings, as perceived discrimination was associated with catastrophizing similarly in NHB and NHW females and was not associated with catastrophizing in NHB or NHW males. Also, associations of discrimination with pain and disability were similar if not stronger in magnitude among NHW compared to NHB females. These findings implicate perceived discrimination as an influential contributor to the pain experiences in women, regardless of race/ethnicity. Discrimination may contribute to the dysregulation of physiological processes associated with chronic, ongoing stress. ${ }^{17,23,26,46}$ Indeed, stressors such 


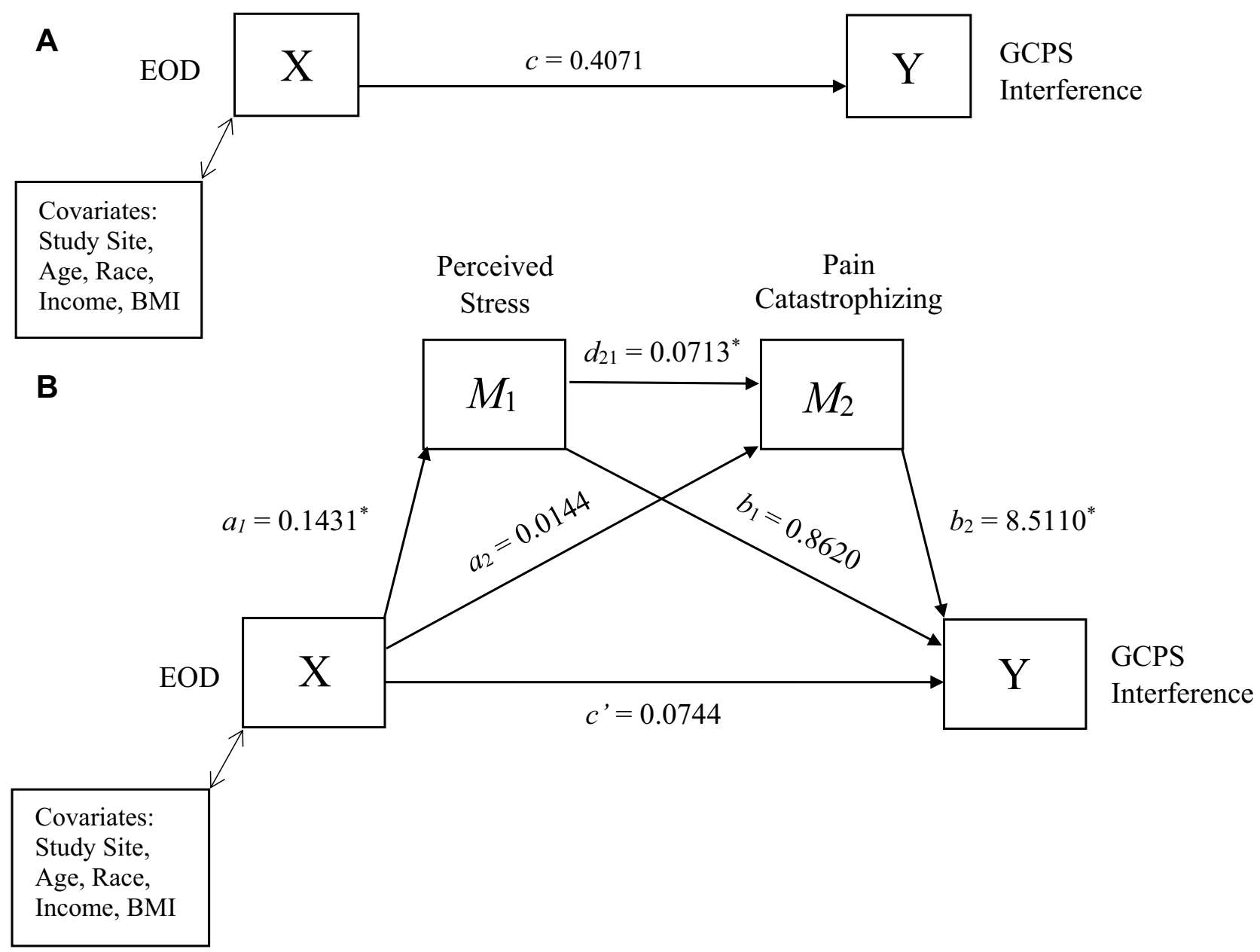

Figure 2 Path diagram showing (A) the total effect of EOD on GCPS Interference and (B) the direct effect and causal paths linking EOD to GCPS interference. *Statistical significance (the bootstrap confidence interval is above or below zero).

Abbreviations: EOD, Everyday discrimination; GCPS Interference, Graded Chronic Pain Scale Interference Scale; BMI, body mass index.

as racial discrimination have been associated with alterations in the hypothalamic-pituitary-adrenal (HPA) axis activity $^{47}$ and cortisol response, ${ }^{48}$ the stress regulating system, greater allostatic load, ${ }^{49}$ presence of circulating pro-inflammatory cytokines, ${ }^{50-52}$ and shorter telomere length. ${ }^{53}$

Interestingly, we found that perceived stress was positively correlated with discrimination in the NHW group, but not among NHB participants. This suggests that the components of stressful experiences assessed by the Perceived Stress Scale are not strongly associated with discrimination among NHB individuals. Indeed, the discrimination experienced by NHB adults is more likely to be chronic and implicit, and therefore may not be consciously appraised as a discreet stressor. In this case, the Perceived Stress Scale, which asks about responses to ongoing demands and stressors, may fail to capture NHB individuals' responses to discrimination and may fail to capture specific situations more common to the Black life experience. Alternatively, NHB individuals may have developed coping strategies for mitigating the effects of discrimination, which would attenuate its impact on perceived stress. Indirect support for this comes from the finding that perceived stress did not differ across race/ethnic groups, despite large differences in discrimination.

In the present study, the relationship between discrimination, perceived stress and pain catastrophizing and outcome variables in mediation analyses were assessed in females only. An interesting but unexpected finding was the lack of relationship between everyday discrimination and outcome variables in males. This was especially 
Table 6 Path Coefficients, Indirect Effects and 95\% BiasCorrected. Bootstrap Confidence Intervals from OLS Regression Predicting Score on Graded Chronic Pain Scale (GCPS) Interference ( $\mathrm{N}=108)$

\begin{tabular}{|l|l|l|l|}
\hline \multirow{2}{*}{ Path } & \multirow{2}{*}{ Estimate } & \multicolumn{2}{|l|}{$\mathbf{9 5 \%} \mathbf{C l}$} \\
\cline { 3 - 4 } & & Lower & Upper \\
\hline Total effect (c) & 0.4071 & -0.0678 & 0.8821 \\
Direct effect (c') & 0.0744 & -0.3748 & 0.5235 \\
$a_{1}$ (EOD $\rightarrow$ Perceived Stress) & $0.1431^{*}$ & 0.0378 & 0.2483 \\
$a_{2}$ (EOD $\rightarrow$ Pain Catastrophizing) & 0.0144 & -0.0036 & 0.0324 \\
$d_{21 \text { (Perceived Stress } \rightarrow \text { Pain Catastrophizing) }}$ & $0.0713^{*}$ & 0.0387 & 0.1040 \\
$b_{1}$ (Perceived Stress $\rightarrow$ GCPS Interference) & 0.8620 & -0.0136 & 1.7375 \\
$b_{2}$ (Pain Catastrophizing $\rightarrow$ GCPS Interference) & $8.5110^{*}$ & 3.6342 & 13.3878 \\
\hline Indirect Effects & & & \\
$a_{1} b_{1}$ & & & \\
$a_{1} d_{21} b_{2}$ & $0.1233^{*}$ & 0.0050 & 0.3598 \\
$a_{2} b_{2}$ & $0.0869^{*}$ & 0.0226 & 0.2312 \\
Total indirect effect & $0.1226^{*}$ & 0.0007 & 0.3096 \\
\hline
\end{tabular}

Notes: *Statistical significance (the bootstrap confidence interval is above or below zero). Models include controls for study site, age, race, income, and body mass index.

notable in NHB males because they reported experiencing the highest rates of discrimination and the poorest clinical pain-related and functional outcomes. In women, we found that perceived stress and pain catastrophizing serially mediate the relationship between everyday discrimination and pain-related and functional outcomes, which resulted in greater clinical pain, greater disability, and higher levels of functional impairment. The analyses revealed a slightly different pattern of findings for each of the 3 outcomes. The serial mediation path in which, experiences of discrimination led to higher perceived stress which led to greater pain catastrophizing was significant for all outcomes. However, the significance of other indirect paths varied across outcomes. For example, the association of discrimination with WOMAC pain was not mediated by perceived stress, while this path was significant for both pain interference and functional performance. In contrast, pain catastrophizing mediated the association of discrimination with WOMAC pain and GCPS pain interference, but not with SPPB performance. The reasons for these differences are not clear, but the findings suggest that the link between discrimination and different pain-related outcomes may be mediated by different psychological factors. Given that perceived gender discrimination in women negatively impacts women's self-esteem ${ }^{54}$ and well-being, ${ }^{55}$ it is possible that experiences of discrimination, including gender-based discrimination may cause women to feel socially excluded or isolated, which could interfere with their ability to benefit from the buffering effects of social support. In turn, this may lead to greater perceived stress and higher use of pain catastrophizing, resulting in poorer pain-related and functional outcomes in women.

These findings have potential clinical implications. Specifically, it may be difficult to directly alter experiences of discrimination; however, our findings suggest that incorporating discrimination as a potential target of interventions could positively impact pain-related outcomes. First, providers should consider assessing discrimination to determine its potential role, particularly in high-risk patients with chronic pain. Among patients who report experiences of discrimination, addressing the potential pathways whereby discrimination might affect pain, for example, through increased perceived stress and greater pain catastrophizing, could be beneficial. This may potentially enhance patients' motivation to address stress, pain catastrophizing or other targets, such that when patients experience episodes of discrimination they can implement pain-coping strategies to reduce the downstream effects of these experiences of discrimination. Essentially, interventions should incorporate strategies that decrease the stress associated with discrimination and limiting the adverse cognitive/affective (eg, pain catastrophizing) processes that may accompany such situations. Finally, strategies to reduce bias and discrimination in the healthcare setting are needed to promote equitable pain medicine. Empowering patients by providing them the tools to advocate for optimal pain care is an important goal, particularly for individuals who are often marginalized by the health system. In addition to incorporating discrimination as a potential treatment target, future studies would benefit from longitudinal designs to determine causal relationships between experiences of discrimination and perceived stress and catastrophizing and their impacts on pain outcomes across race and sex. Studies investigating the impact on gender discrimination on pain are needed as well as research investigating the influence of discrimination on pain catastrophizing.

These findings should be interpreted in light of the study's limitations. First, this is a cross-sectional study and was not originally designed as a serial mediation model; therefore, it is unclear the directionality of the 


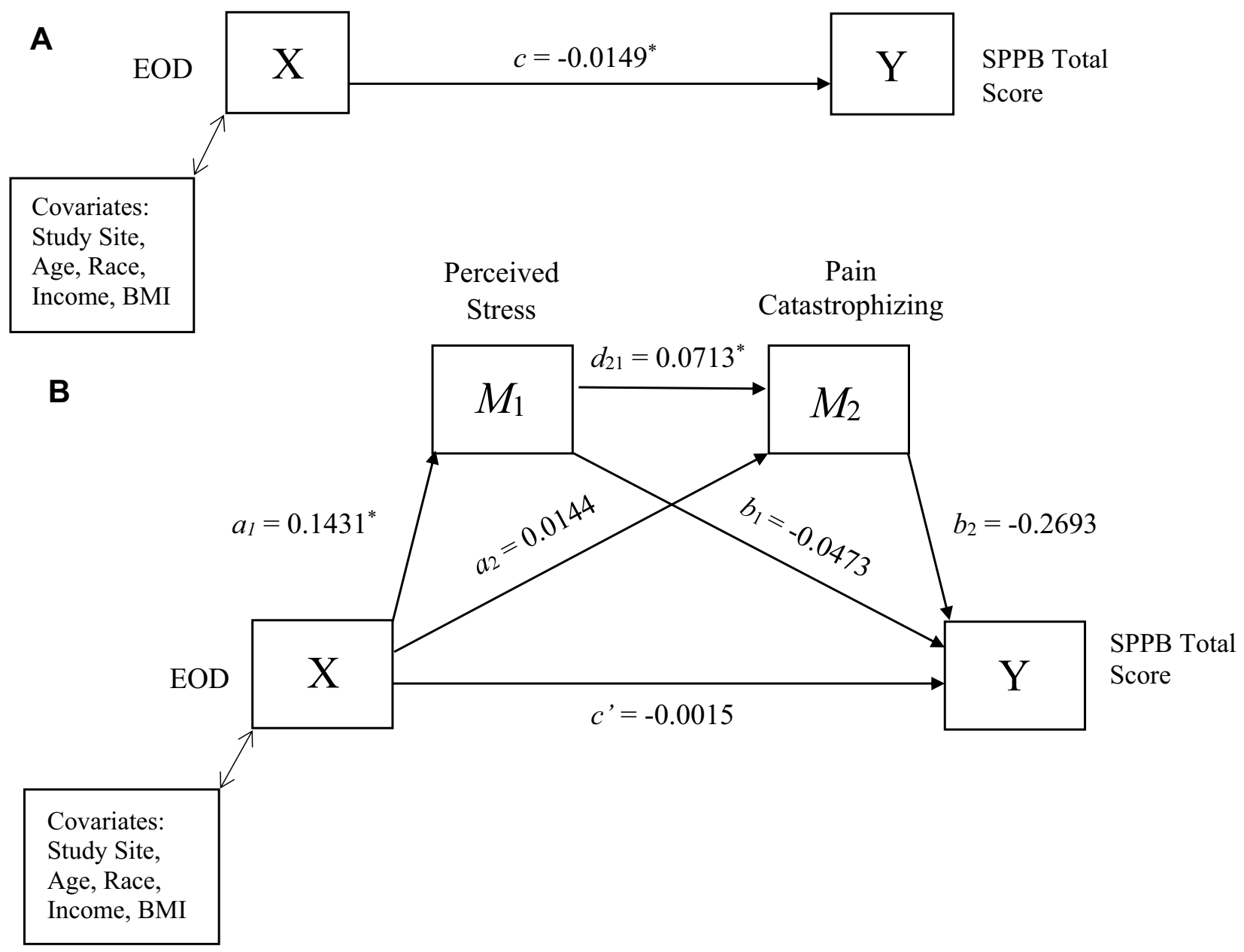

Figure 3 Path diagram showing (A) the total effect of EOD on SPPB Total and (B) the direct effect and causal paths linking EOD to SPPB Total. *Statistical significance (the bootstrap confidence interval is above or below zero).

Abbreviations: EOD, Everyday discrimination; SPPB, Short Performance Physical Battery; BMI, body mass index.

relationship between perceived stress and pain catastrophizing. It is likely a bidirectional relationship where change in one variable impacts the other and vice versa. Therefore, future studies should be designed to test the order of the mediators. Also, additional psychological variables such as anxiety and depression were not included in the model, and these may have more of an impact on outcomes for males. Lastly, it is not clear whether these findings generalize to a younger cohort (eg, 18-44 years of age), to individuals with different pain conditions, or even other cohorts of NHB participants.

The current study has several strengths. This is the first study to examine this serial mediation model and to assess the intersection between race and sex in patients with chronic knee pain. The study includes a large sample that is ethnically/racially diverse. The study used established measures to assess experiences of discrimination, as well as psychosocial and outcome variables.

In sum, the results of the current study suggest NHB males and females experience higher rates of racial discrimination compared to their NHW counterparts. Our findings also suggest that perceived stress and pain catastrophizing serially mediate the relationship between everyday discrimination and poor pain-related outcomes in women. These findings underscore the importance of perceived stress and pain catastrophizing in understanding the complex relationship between everyday discrimination and knee OA-related clinical pain, disability and physical functioning outcomes. These results may have implications for the management of perceived stress and pain catastrophizing as a means to reduce the negative impact of experiences of discrimination in relation to the experience of knee pain. 
Table 7 Path Coefficients, Indirect Effects and 95\% BiasCorrected Bootstrap Confidence Intervals from OLS Regression Predicting Score on Short Performance Physical Battery (SPPB) Total Score ( $\mathrm{N}=108)$

\begin{tabular}{|l|l|l|l|}
\hline \multirow{2}{*}{ Path } & \multirow{2}{*}{ Estimate } & \multicolumn{2}{|l|}{$95 \%$ CI } \\
\cline { 3 - 4 } & & Lower & Upper \\
\hline Total effect $(c)$ & $-0.0149^{*}$ & $-0.041 \mathrm{I}$ & -0.0113 \\
Direct effect (c') & -0.0015 & -0.0278 & 0.0248 \\
$a_{1}$ (EOD $\rightarrow$ Perceived Stress) & $0.1431^{*}$ & 0.0387 & 0.2483 \\
$a_{2}$ (EOD $\rightarrow$ Pain Catastrophizing) & 0.0144 & -0.0036 & 0.0324 \\
$d_{21}$ (Perceived Stress $\rightarrow$ Pain Catastrophizing) & $0.0713^{*}$ & 0.0387 & 0.1040 \\
$b_{1}$ (Perceived Stress $\rightarrow$ SPPB) & -0.0473 & -0.0986 & 0.0040 \\
$b_{2}$ (Pain Catastrophizing $\rightarrow$ SPPB) & -0.2693 & -0.5550 & 0.0164 \\
\hline Indirect Effects & & & \\
$a_{1} b_{1}$ & $-0.0068^{*}$ & -0.0197 & -0.0001 \\
$a_{1} d_{2 \mid} b_{2}$ & $-0.0027^{*}$ & -0.0093 & -0.0002 \\
$a_{2} b_{2}$ & -0.0039 & -0.0126 & 0.0002 \\
Total indirect effect & $-0.0134^{*}$ & -0.0298 & -0.0037 \\
\hline
\end{tabular}

Notes: *Statistical significance (the bootstrap confidence interval is above or below zero). Models include controls for site, age, race, income, and body mass index.

\section{Funding}

Research funding and support provided by NIH/NIA Grants R37AG033906 (RBF) and R01AG054370 (KTS); UF CTSA Grant UL1TR001427 and UAB CTSA Grant UL1TR001417 from the NIH Center for Advancing Translational Sciences; 1K22NS102334 and 1P30AG059297 provided to the University of Florida and McKnight Brain Institute Career Development Award (ELT), minority supplement provided to the University of Florida (JSC), NIH Training Grants TL1TR001418 provided to the University of Alabama at Birmingham (KAT); NIH/NIA Grant R00AG052642 provided to the University of Florida (EJB).

\section{Disclosure}

The authors declare no conflicts of interest.

\section{References}

1. Cisternas MG, Murphy L, Sacks JJ, Solomon DH, Pasta DJ, Helmick CG. Alternative methods for defining osteoarthritis and the impact on estimating prevalence in a US population-based survey. Arthritis Care Res (Hoboken). 2016;68(5):574-580. doi:10.1002/acr.22721

2. Mokdad AH, Ballestros K, Echko M, et al. The state of US health, 1990-2016: burden of diseases, injuries, and risk factors among US states. JAMA. 2018;319(14):1444-1472. doi:10.1001/jama.2018.0158

3. Park J, Mendy A, Vieira ER. Various types of arthritis in the united states: prevalence and age-related trends from 1999 to 2014. Am J Public Health. 2018;108(2):256-258. doi:10.2105/AJPH.2017.304179

4. Lawrence RC, Felson DT, Helmick CG, et al. Estimates of the prevalence of arthritis and other rheumatic conditions in the United States: part II. Arthritis Rheumatol. 2008;58(1):26-35. doi:10.1002/ art.23176
5. Cross M, Smith E, Hoy D, et al. The global burden of hip and knee osteoarthritis: estimates from the global burden of disease 2010 study. Ann Rheum Dis. 2014;73(7):1323-1330. doi:10.1136/annrheumdis2013-204763

6. Litwic A, Edwards MH, Dennison EM, Cooper C. Epidemiology and burden of osteoarthritis. Br Med Bull. 2013;105(1):185-199. doi: $10.1093 / \mathrm{bmb} / \mathrm{lds} 038$

7. Palazzo C, Nguyen C, Lefevre-Colau -M-M, Rannou F, Poiraudeau S. Risk factors and burden of osteoarthritis. Ann Phys Rehabil Med. 2016;59(3):134-138. doi:10.1016/j.rehab.2016.01.006

8. Losina E, Weinstein AM, Reichmann WM, et al. Lifetime risk and age at diagnosis of symptomatic knee osteoarthritis in the US. Arthritis Care Res (Hoboken). 2013;65(5):703-711. doi:10.1002/ acr.21898

9. Srikanth VK, Fryer JL, Zhai G, Winzenberg TM, Hosmer D, Jones G. A meta-analysis of sex differences prevalence, incidence and severity of osteoarthritis. Osteoarthritis Cartilage. 2005;13(9):769-781. doi:10.1016/j.joca.2005.04.014

10. Jordan JM, Helmick CG, Renner JB, et al. Prevalence of knee symptoms and radiographic and symptomatic knee osteoarthritis in African Americans and Caucasians: the Johnston County Osteoarthritis Project. J Rheumatol. 2007;34(1):172-180.

11. Parmelee PA, Harralson TL, McPherron JA, DeCoster J, Schumacher HR. Pain, disability, and depression in osteoarthritis: effects of race and sex. J Aging Health. 2012;24(1):168-187. doi: $10.1177 / 0898264311410425$

12. Collins JE, Deshpande BR, Katz JN, Losina E. Race-and sex-specific incidence rates and predictors of total knee arthroplasty: seven-year data from the Osteoarthritis Initiative. Arthritis Care Res (Hoboken). 2016;68(7):965-973. doi:10.1002/acr.22771

13. Vaughn IA, Terry EL, Bartley EJ, Schaefer N, Fillingim RB. Racialethnic differences in osteoarthritis pain and disability: a meta-analysis. J Pain. 2019;20(6):629-644.

14. Golightly YM, Dominick KL. Racial variations in self-reported osteoarthritis symptom severity among veterans. Aging Clin Exp Res. 2005;17(4):264-269. doi:10.1007/BF03324608

15. Cruz-Almeida Y, Sibille KT, Goodin BR, et al. Racial and ethnic differences in older adults with knee osteoarthritis. Arthritis Rheumatol. 2014;66(7):1800-1810. doi:10.1002/art.38620

16. Cano A, Mayo A, Ventimiglia M. Coping, pain severity, interference, and disability: the potential mediating and moderating roles of race and education. $J$ Pain. 2006;7(7):459-468. doi:10.1016/j. jpain.2006.01.445

17. Walker JL, Harrison TC, Brown A, Thorpe RJ Jr, Szanton SL. Factors associated with disability among middle-aged and older African American women with osteoarthritis. Disabil Health J. 2016;9(3):510-517. doi:10.1016/j.dhjo.2016.02.004

18. Burns R, Graney MJ, Lummus AC, Nichols LO, Martindale-Adams J. Differences of self-reported osteoarthritis disability and race. J Natl Med Assoc. 2007;99(9):1046-1051.

19. Luong M-LN, Cleveland RJ, Nyrop KA, Callahan LF. Social determinants and osteoarthritis outcomes. Aging Health. 2012;8 (4):413-437. doi:10.2217/ahe.12.43

20. Krieger N, Smith K, Naishadham D, Hartman C, Barbeau EM. Experiences of discrimination: validity and reliability of a self-report measure for population health research on racism and health. Soc Sci Med. 2005;61(7):1576-1596. doi:10.1016/j.socscimed.2005.03.006

21. Pascoe EA, Smart Richman L. Perceived discrimination and health: a meta-analytic review. Psychol Bull. 2009;135(4):531. doi:10.1037/ a0016059

22. Fiske ST. What we know now about bias and intergroup conflict, the problem of the century. Curr Dir Psychol Sci. 2002;11(4):123-128. doi:10.1111/1467-8721.00183

23. Potter LN, Brondolo E, Smyth JM. Biopsychosocial correlates of discrimination in daily life: a review. Stigma Health. 2019;4(1):38. doi: $10.1037 /$ sah0000120 
24. McIlvane JM, Baker TA, Mingo CA. Racial differences in arthritis-related stress, chronic life stress, and depressive symptoms among women with arthritis: a contextual perspective. J Gerontology Series B. 2008;63(5):S320-S327. doi:10.1093/geronb/63.5.S320

25. Goodin BR, Pham QT, Glover TL, et al. Perceived racial discrimination, but not mistrust of medical researchers, predicts the heat pain tolerance of African Americans with symptomatic knee osteoarthritis. Health Psychol. 2013;32(11):1117-1126. doi:10.1037/a0031592

26. Brown TT, Partanen J, Chuong L, Villaverde V, Griffin AC, Mendelson A. Discrimination hurts: the effect of discrimination on the development of chronic pain. Soc Sci Med. 2018;204:1-8. doi:10.1016/j.socscimed.2018.03.015

27. Burgess DJ, Grill J, Noorbaloochi S, et al. The effect of perceived racial discrimination on bodily pain among older African American men. Pain Med. 2009;10(8):1341-1352. doi:10.1111/j.1526-4637.2009.00742.x

28. Edwards RR. The association of perceived discrimination with low back pain. J Behav Med. 2008;31(5):379. doi:10.1007/s10865-008-9160-9

29. Keefe FJ, Lefebvre JC, Kerns RD, et al. Understanding the adoption of arthritis self-management: stages of change profiles among arthritis patients. Pain. 2000;87(3):303-313. doi:10.1016/S0304-3959(00)00294-3

30. Waugh OC, Byrne DG, Nicholas MK. Internalized stigma in people living with chronic pain. J Pain. 2014;15(5):e551-550. () doi:10.1016/j.jpain.2014.02.001

31. Altman R, Asch E, Bloch D, et al. Development of criteria for the classification and reporting of osteoarthritis: classification of osteoarthritis of the knee. Arthritis Rheumatol. 1986;29(8):1039-1049. doi:10.1002/art.1780290816

32. Roux CH, Saraux A, Mazieres B, et al. Screening for hip and knee osteoarthritis in the general population: predictive value of a questionnaire and prevalence estimates. Ann Rheum Dis. 2008;67 (10):1406-1411. doi:10.1136/ard.2007.075952

33. Kraus VB, Blanco FJ, Englund M, Karsdal MA, Lohmander LS. Call for standardized definitions of osteoarthritis and risk stratification for clinical trials and clinical use. Osteoarthritis Cartilage. 2015;23 (8):1233-1241. doi:10.1016/j.joca.2015.03.036

34. Bellamy N, Buchanan WW, Goldsmith CH, Campbell J, Stitt LW. Validation study of WOMAC: a health status instrument for measuring clinically important patient relevant outcomes to antirheumatic drug therapy in patients with osteoarthritis of the hip or knee $J$ Rheumatol. 1988;15(12):1833-1840.

35. Theiler R, Spielberger J, Bischoff H, Bellamy N, Huber J, Kroesen S. Clinical evaluation of the WOMAC 3.0 OA Index in numeric rating scale format using a computerized touch screen version. Osteoarthritis Cartilage. 2002;10(6):479-481. doi:10.1053/joca.2002.0807

36. Von Korff M, Ormel J, Keefe FJ, Dworkin SF. Grading the severity of chronic pain. Pain. 1992;50(2):133-149. doi:10.1016/0304-3959(92)90154-4

37. Guralnik JM, Simonsick EM, Ferrucci L, et al. A short physical performance battery assessing lower extremity function: association with self-reported disability and prediction of mortality and nursing home admission. J Gerontol. 1994;49(2):M85-M94. doi:10.1093/geronj/49.2.M85

38. Cohen S, Kamarck T, Mermelstein R. A global measure of perceived stress. J Health Soc Behav. 1983;24(4):385-396. doi:10.2307/2136404

39. Cohen S, Kamarck T, Mermelstein R. Perceived stress scale. Measuring stress: a guide for health and social scientists. 1994;235-283.
40. Rosenstiel AK, Keefe FJ. The use of coping strategies in chronic low back pain patients: relationship to patient characteristics and current adjustment. Pain. 1983;17(1):33-44. doi:10.1016/0304-3959(83)90125-2

41. Jensen MP, Keefe FJ, Lefebvre JC, Romano JM, Turner JA. One-and two-item measures of pain beliefs and coping strategies. Pain. 2003;104(3):453-469. doi:10.1016/S0304-3959(03)00076-9

42. Hayes AF. PROCESS: a versatile computational tool for observed variable mediation, moderation, and conditional process modeling. 2012.

43. Hayes AF. Introduction to Mediation, Moderation, and Conditional Process Analysis: a Regression-Based Approach. New York, NY: The Guiford Press; 2013.

44. Gee GC, Spencer MS, Chen J, Takeuchi D. A nationwide study of discrimination and chronic health conditions among Asian Americans. Am J Public Health. 2007;97(7):1275-1282. doi:10.2105/AJPH.2006.091827

45. Anderson KO, Green CR, Payne R. Racial and ethnic disparities in pain: causes and consequences of unequal care. J Pain. 2009;10 (12):1187-1204. doi:10.1016/j.jpain.2009.10.002

46. Berger M, Sarnyai Z. "More than skin deep": stress neurobiology and mental health consequences of racial discrimination. Stress. 2015;18 (1):1-10. doi:10.3109/10253890.2014.989204

47. Busse D, Yim IS, Campos B, Marshburn CK. Discrimination and the HPA axis: current evidence and future directions. J Behav Med. 2017;40(4):539-552. doi:10.1007/s10865-017-9830-6

48. Adam EK, Heissel JA, Zeiders KH, et al. Developmental histories of perceived racial discrimination and diurnal cortisol profiles in adulthood: a 20-year prospective study. Psychoneuroendocrinology. 2015;62:279-291. doi:10.1016/j.psyneuen.2015.08.018

49. Allen AM, Thomas MD, Michaels EK, et al. Racial discrimination, educational attainment, and biological dysregulation among midlife African American women. Psychoneuroendocrinology. 2019;99: 225-235. doi:10.1016/j.psyneuen.2018.09.001

50. Stepanikova I, Bateman LB, Oates GR. Systemic inflammation in midlife: race, socioeconomic status, and perceived discrimination. $\mathrm{Am}$ J Prev Med. 2017;52(1):S63-S76. doi:10.1016/j.amepre.2016.09.026

51. Lewis TT, Aiello AE, Leurgans S, Kelly J, Barnes LL. Self-reported experiences of everyday discrimination are associated with elevated C-reactive protein levels in older African-American adults. Brain Behav Immun. 2010;24(3):438-443. doi:10.1016/j.bbi.2009.11.011

52. Van Dyke ME, Vaccarino V, Dunbar SB, et al. Socioeconomic status discrimination and C-reactive protein in African-American and White adults. Psychoneuroendocrinology. 2017;82:9-16. doi:10.1016/j. psyneuen.2017.04.009

53. Lee DB, Kim ES, Neblett EW Jr. The link between discrimination and telomere length in African American adults. Health Psychol. 2017;36(5):458. doi:10.1037/hea0000450

54. Schmitt MT, Branscombe NR, Postmes T. Women's emotional responses to the pervasiveness of gender discrimination. Eur J Soc Psychol. 2003;33(3):297-312. doi:10.1002/ejsp.147

55. Schmitt MT, Branscombe NR, Kobrynowicz D, Owen S. Perceiving discrimination against one's gender group has different implications for well-being in women and men. Personality Social Psychol Bull. 2002;28(2):197-210. doi:10.1177/0146167202282006

Journal of Pain Research

Dovepress

\section{Publish your work in this journal}

The Journal of Pain Research is an international, peer reviewed, open access, online journal that welcomes laboratory and clinical findings in the fields of pain research and the prevention and management of pain. Original research, reviews, symposium reports, hypothesis formation and commentaries are all considered for publication. The manuscript

management system is completely online and includes a very quick and fair peer-review system, which is all easy to use. Visit http:// www.dovepress.com/testimonials.php to read real quotes from published authors. 